\title{
ESTUDIO DE PREFACTIBILIDAD PARA LA ORGANIZACIÓN COMUNITARIA EN LA CONSTRUCCIÓN DE UNA CENTRAL DE CAFÉ CEREZA EN EL MUNICIPIO DE LA PALMA, CUNDINAMARCA, COMO OPCIÓN DE PAZ
}

\author{
PRE-FEASIBILITY STUDY FOR THE COMMUNITY \\ ORGANIZATION IN THE CONSTRUCTION \\ OF A COFFEE PLANT IN THE MUNICIPALITY OF \\ LA PALMA, CUNDINAMARCA, \\ AS A PEACE OPTION
}

Néstor Javier Robayo Rojas*

Nubia Esperanza Suárez Suárez**

César Andrés Pinilla Herrera***

* Ingeniero Agrónomo de la Universidad Nacional de Colombia. MBA. Docente Investigador de la Universidad Santo Tomás (Colombia). Correo electrónico:nestorrobayo@ustadistancia.edu.co

** Ingeniera Agrónoma de la Universidad Nacional de Colombia, MBA de la Escuela Europea de Negocios, Estudiante Doctorado en proyectos de la Universidad Internacional Iberoamericana Unini Funiber. Docente Investigador de la Universidad Santo Tomás (Colombia).

Correo electrónico: nubiasuarez@ustadistancia.edu.co

*** Estudiante de Administración de Empresas Agropecuarias de la Universidad Santo Tomás (Colombia). Correo electrónico: cesarpinillah@ustadistancia.edu.co 
Estudio de prefactibilidad para la organización comunitaria en la construcción de una central de café cereza en el municipio de La Palma, Cundinamarca, como opción de paz

\section{RESUMEN}

El municipio de la Palma, Cundinamarca tiene como base de su economía la agricultura y la ganadería, siendo el cultivo de café el motor de su desarrollo. La Asociación de Caficultores de la Palma "Asoparibari" ha organizado a familias de la zona, buscando mejorar los procesos de organización comunitaria, productividad y sostenibilidad cafetera. Por otro lado, la Federación Nacional de Cafeteros ha venido impulsados proyectos para la construcción de centrales de beneficio, con lo cual busca mejorar la calidad del grano y el manejo de subproductos. Uno de esos proyectos está enfocado para ser administrado por Asoparibari.

Teniendo en cuenta que el municipio de la Palma tiene I 204 hectáreas cultivadas con café y se concentra principalmente en una zona geográfica, la asociación Asoparibari ha decidido orientar sus esfuerzos en buscar recursos para la construcción y puesta en marcha de una central de beneficio que favorezca cerca de 100 familias de la zona. Sin embargo, surgen dudas como ¿Se justifica la construcción de la central de beneficio? ¿Está en capacidad la asociación de operar la central de beneficio? ¿Cuentan con la suficiente capacidad logística para ejecutar el proyecto? ¿Están los asociados dispuestos a llevar su café a la asociación? ¿Hay suficiente credibilidad en las directivas de la asociación? Estas y más preguntas surgen al momento de pensar en este proyecto, ya que, si no se hace un estudio previo, el proyecto se puede convertir en una gran dificultad para la asociación y un desperdicio de recursos tanto económicos como humanos.

Este artículo expone el estudio de prefactibilidad, que determinó la viabilidad técnica para la construcción de una central de café cereza en el municipio de La Palma Cundinamarca, identificando debilidades y fortalezas que permiten realizar recomendaciones para mejorar procesos organizacionales a la asociación de productores Asoparibari sobre la pertinencia de continuar o no con el proceso para la construcción de esta central de beneficio, aplicando la metodología del Índice de Capacidades Organizacionales en Comunidades Cafeteras, ICOCC.

Palabras claves: índice de capacidades organizacionales, organización comunitaria, productividad y sostenibilidad cafetera.

\section{ABSTRACT}

The municipality of La Palma, Cundinamarca is based on agriculture and livestock farming, with coffee growing the engine of development. The Association of Coffee Growers of the Palm" 
Asoparibari has organized families in the area, seeking to improve the processes of community organization, productivity and coffee sustainability. On the other hand, the National Federation of Coffee Growers has been promoting projects for the construction of coffee production centers seeking to improve the quality of grain and the management of by-products. One such project is focused on being managed by Asoparibari.

Since the municipality of La Palma has 1204 hectares cultivated with coffee and concentrates mainly in a geographical area, the association Asoparibari has decided to orient its efforts in finding resources for the construction and start-up of a profit center that favors near of 100 families in the area. However, doubts arise as to whether the construction of the profit center is justified? Are the association operating the profit center? Do they have sufficient logistical capacity to carry out the project? Are the partners willing to bring their coffee to the association? Is there sufficient credibility in the association? These and more questions arise now of thinking about this project, since, if a previous study is not done, the project can become a great difficulty for the association and a waste of both economic and human resources.

In conclusion, this project will show the prefeasibility study that seeks to determine the Technical Viability, for the construction of a cherry coffee plant in the municipality of La Palma Cundinamarca, identifying weaknesses and strengths that will allow to make recommendations to improve organizational processes to the association of Asoparibari producers on the pertinence of continuing or not with the process for the construction of this profit center, applying the methodology of the Organizational Capacities Index in Coffee Communities, ICOCC.

Key Words: index of capacities organizations, community organization, productivity and coffee sustainability 


\section{INTRODUCCIÓN}

El municipio de la Palma se encuentra ubicado en el noroccidente de Cundinamarca, en la provincia del Rionegro, a $150 \mathrm{~km}$ de distancia de la ciudad de Bogotá. El municipio tiene como base de su economía la agricultura y la ganadería, siendo el cultivo de café el motor de desarrollo, que lo posiciona en los primeros lugares de producción del departamento durante muchos años.

Para finales de los años 90 y principios de la década del 2000, La Palma afrontó un gran conflicto armado. La lucha por el territorio ocasionó un desplazamiento masivo. Los campesinos, ubicados en la zona rural, fueron sacados de sus tierras y obligados a marcharse a otras zonas del país como Pacho, Zipaquirá y Bogotá. Este conflicto ocasionó que el municipio de La Palma fuera uno de los más afectados por la violencia en el departamento.

La Federación Nacional de Cafeteros de Colombia (FNC) ha apoyado al sector ruraly en especial a las familias caficultoras de la zona, por medio de proyectos de orden social y económico. De esta forma, un grupo de caficultores del municipio de La Palma, buscando mejores alternativas para la producción y comercialización del café, fundó la Asociación de Caficultores de la Palma “Asoparibari”. Alrededor de esta asociación se han organizado aproximadamente 100 familias de la zona más cafetera del municipio, con el fin de mejorar los procesos de ordenamiento comunitarios para lograr obtener apoyo de las diferentes entidades de cooperación.

Desde hace varios años, la FNC ha venido impulsando proyectos para la construcción de centrales de beneficio del café que permitan concentrar todo el proceso de despulpado, fermentado y lavado en un mismo lugar. Al aplicar un proceso estandarizado en un mismo sitio, se pueda mejorar la calidad del café y a su vez concentrar el manejo de los subproductos conforme a las indicaciones de las autoridades ambientales. De esta manera se podría mejorar la calidad del café que, en su gran mayoría, se deteriora por los inadecuados procesos de beneficio y secado, al tiempo que se disminuyen los vertimientos en cada una de las fincas de los caficultores.

La asociación Asoparibari tiene un terreno donde se planea construir la central. Además, en una reunión de asociados, la gran mayoría estuvo de acuerdo en poner en marcha las gestiones para ejecutar este proyecto y que sea operado por dicha asociación. Sin embargo, surgen dudas como las siguientes: ¿Se justifica la construcción de la central de beneficio? ¿Está en capacidad la asociación de operar la central de beneficio? ¿Cuentan con la 
suficiente capacidad logística para ejecutar el proyecto? ¿Están los asociados dispuestos a llevar su café a la asociación? ¿Hay suficiente credibilidad en las directivas de la asociación? Estas y más preguntas surgen al momento de pensar en este proyecto, ya que, si no se hace un estudio previo, el proyecto se puede convertir en una gran dificultad para la asociación y un desperdicio de recursos económicos y humanos.

Este proyecto tiene como objetivo principal obtener información en las diferentes dimensiones de la sostenibilidad; dicha información será el insumo básico para la toma de decisiones respecto a la construcción y puesta en marcha de una Central de Beneficio de Café. La propuesta tiene un alcance que va más allá de la construcción física de la Central, incluyendo los aspectos técnicos externos, sociales, ambientales y económicos que rodean el proyecto y que son fundamentales para que este tipo de iniciativas lleguen a buen término.

El objeto de esta investigación es efectuar un estudio de prefactibilidad para la construcción de una central de café cereza en el municipio de La Palma Cundinamarca, identificando debilidades y fortalezas de la Asociación Asoparibari para establecer la pertinencia operativa de la administración de la central de beneficio por parte de la asociación, aplicando las técnicas de Índice de Viabilidad Técnica (IVT) y el Índice de Capacidades Organizacionales de Comunidades Cafeteras (ICOCC) desarrollados por Cenicafé.

Con esa investigación se espera determinar la viabilidad con la que se ejecutará la central de beneficio para tener la probabilidad de ofrecer café de alta calidad a gran escala, mediante un proceso altamente ecológico. Brindando a los caficultores asociados la posibilidad de mejores precios en el mercado, permitiendo procesar café a un precio equivalente al que se paga en la zona central cafetera.

\section{CONTENIDO}

El campo colombiano contribuye de manera importante al desarrollo económico y social del país. En efecto, el 84,7 \% del territorio colombiano está conformado por municipios totalmente rurales, y según la Misión para la Transformación del Campo, el 30,4 \% de la población colombiana vive en zonas rurales (Garcia y Soto, 2012, citados por Federacion Nacional de Cafeteros de Colombia, 2014). Por su parte, el sector agropecuario aporta en promedio 6,1 \% del PIB total y genera el 16,3\% del empleo del país (DANE, 2017). Además, el campo cuenta con más de $\mathbf{4 2}$ millones de hectáreas aptas para las actividades agropecuarias y forestales $y$ es la fuente de los recursos naturales que ofrecen ventajas comparativas 
para el país como los hidrocarburos, los minerales y la biodiversidad.

Por décadas, la cultura cafetera ha forjado el entorno social y económico del país, no solo en el plano de desarrollo rural, sino también como una ventana de inserción de la economía local en el contexto internacional y fuente importante de divisas, que contribuyó de manera decisiva al crecimiento y modernización de la industria y comercio nacional (Urueña, 20I3).

El cultivo del café en Colombia se desarrolla principalmente en las vertientes de la cordillera de los andes con influencia de los océanos y la Amazonía, lo cual genera condiciones climáticas y regímenes de lluvia excepcionales, que le permiten al país cosechar café durante todos los meses del año. El origen volcánico de los suelos y las altas alturas en las que se produce café en Colombia, que se derivan de su cercanía con la línea ecuatorial, le confieren atributos balanceados al café colombiano. El café colombiano es suave, de taza limpia, con acidez y cuerpo medio/alto, $y$ aroma pronunciado y completo.

La caficultura del departamento de Cundinamarca es desarrollada por 31.315 caficultores, cuyas familias se agrupan en 69 municipios (que equivalen al $60 \%$ de los municipios del departamento); constituyéndose en una de las principales fuentes de ingreso para más de 123 mil personas en el sector rural. Por ser intensiva en mano de obra, se generan más de 100.000 empleos entre directos e indirectos, siendo una de las actividades agropecuarias que más empleos genera en el departamento. La caficultura de Cundinamarca es una actividad de pequeños propietarios, el tamaño promedio de área en café por caficultor es de I,2 hectáreas (Arcila, Farfán Valencia, Moreno, Salazar y Hincapié, 2007).

Actualmente la caficultura del municipio de La Palma se desarrolla en 1204 hectáreas, trabajadas por 1196 caficultores, siendo uno de los cultivos más importantes del municipio, pues lo ubica en el quinto lugar de producción en el departamento. La estructura cafetera del municipio de la Palma está principalmente en cafetales tecnificados jóvenes con un $83 \%$ del área total y un $17 \%$ entre caficultura envejecida $y$ tradicional.

La Federación Nacional de Cafeteros de Colombia, a través de Centro Nacional de Investigaciones del Café (Cenicafe), ha desarrollado investigaciones que han generado estrategias en 
busca de la transformación de los beneficiaderostradicionales' en beneficiaderos ecológicos ${ }^{2}$.

Recientes estudios afirman que la organización comunitaria (como una expresión de la asociatividad) es un elemento importante, no solo en el incremento de activos económicos familiares para pequeños productores, sino que a su vez es un factor determinante para que la comunidad pueda incidir y tener mayor participación en la resolución de problemáticas que la afectan (Ministerio de Agricultura y Desarrollo Rural, 2003). En ese orden de ideas, a mayor organización comunitaria, mayores posibilidades de soluciones y cambios efectivos que respondan a necesidades e intereses de una comunidad particular. Razón por la cual, la promoción de la asociatividad de pequeños productores, debe consolidarse como área temática a priorizar en investigaciones y proyectos de implementación.

La asociación de caficultores del municipio de La Palma Asoparibari, conelapoyodel Comité Departamental de Cafeteros de Cundinamarca, la Gobernación de Cundinamarcayel Ministerio de Agricultura, han visto la necesidad que la comunidad cafetera implemente una Central de Beneficio de Café, que permita adoptarlas mejores tecnologías para la transformación de café cereza a café pergamino seco, introduciendo conceptos ecológicos en el proceso de beneficio del café; que responda a exigencias legales y ambientales; contribuya a la homogenización de la calidad del producto; minimice los costos de transformación de los beneficiaderos contaminantes a una microcentral con criterios de anejo ecológico y, por lo tanto, mejore los ingresos de los caficultores, sus familias y la zona cafetera.

Una Central de Beneficio de Café consiste en una instalación de carácter industrial, normalmente de gran tamaño, al que llega café en cereza de diferentes caficultores a quienes se les reconoce un pago por su producto de acuerdo con la calidad y cantidad. Dentro de las instalaciones de la central de beneficio tiene lugar la transformación del café en cereza a café pergamino seco (c.p.s), a través de una

1 Instalación para realizar el proceso de despulpado y lavado del café donde se utilizan grandes cantidades de agua con el fin de retirar el mucilago que recubre la semilla del café.

2 Se puede definir el proceso de beneficio ecológico del café por vía húmeda como "el conjunto de operaciones realizadas para transformar el café cereza en café pergamino seco, conservando la calidad exigida por las normas de comercialización, evitando perdidas del producto y eliminando procesos innecesarios, lográndose además, el aprovechamiento de los subproductos lo cual representa el mayor ingreso económico para el caficultor y la mínima alteración del agua estrictamente necesaria para el beneficio". 
serie de equipos que hacen básicamente el despulpado, la remoción del mucílago, varias clasificaciones y el secado. El café se comercializa en el estado de café pergamino seco, con una humedad entre el 10 y $12 \%$ base húmeda (Centro Nacional de Investigaciones del Café - Cenicafé, 2016).

La transformación del café cereza en café pergamino seco en centrales de beneficio tiene las siguientes fortalezas:

\section{- Solamente se debe producir buen café en cereza}

- Se tiene mayor control sobre el proceso (consistencia)

- Se tiene mayor control sobre el impacto ambiental

- Mejor costo de oportunidad para los caficultores

- Mejor aprovechamiento de la economía de escala

Todo lo anterior corresponde al ámbito técnico; sin embargo, el componente social es muy importante dentro de cualquier proceso de adopción tecnológica y sobre todo debe verse desde la organización comunitaria y la asociatividad, ya que esto le permitirá tener un mayor respaldo para el desarrollo de proyectos y la gestión de recursos.

En este sentido, debe realizarse un proceso de análisis técnico y organizacional a la asociación Asoparibari, de tal manera que permita verificar la viabilidad para la construcción de la central de beneficio de café cereza. Para realizar este análisis Cenicafé desarrolló el Índice de Viabilidad Técnica (IVT) y el Índice de Capacidades Organizacionales de Comunidades Cafeteras (ICOCC). El Índice de Viabilidad Técnica se determina mediante la realización de una encuesta a los potenciales beneficiarios del proyecto de construcción de la central de café cereza, a los cuales se les formula una seria de preguntas relacionadas con la producción de café en sus fincas, el estado de la infraestructura productiva, el estado de las vías, la distancia al centro de beneficio $y$ otras preguntas que permiten cuantificar si, desde el punto de vista técnico, existe viabilidad o no para la construcción de la central.

El Índice de Capacidades Organizacionales de Comunidades Cafeteras - ICOCC es una herramienta de diagnóstico, y como tal brinda información sobre la situación "actual" de una organización. En esa medida, se pueden identificar las debilidades y fortalezas de una organización. El índice está compuesto por 
una medición objetiva expresada en números, en una escala prefijada, de los niveles de desarrollo de una organización de productores. Por tanto, la información que brinda el Índice de Capacidades Organizacionales de Comunidades Cafeteras - ICOCC, es de tipo cuantitativo, y posibilita medir el grado de desempeño de las organizaciones.

El Índice de Capacidades Organizacionales de Comunidades Cafeteras - ICOCC se obtiene a partir de la aplicación de un instrumento (encuesta), estructurado bajo las áreas relevantes de desarrollo de una organización (Espinel, 2010). Facilita la formulación y gestión de acciones que contribuyan en el desempeño organizacional, permitiendo priorizar áreas a intervenir. De igual forma, es un insumo para la formulación de indicadores de planes de acción, en tanto facilita el desarrollo de planes de monitoreo y seguimiento.

Por otra parte, esta herramienta contribuye a que la identificación de debilidades y fortalezas de una organización se haga en conjunto con los asociados y directivos, y estos puedan determinar la proyección de la organización, y así ser artífices de su propio desarrollo. Una planificación participativa de acciones a realizar en una organización facilita el cambio de actitud y ratificación de compromiso de los involucrados.
Estudios recientes dan cuenta de que la organización comunitaria es un elemento importante, no solo en el incremento de activos económicos familiares, sino que a su vez es un factor determinante para que la comunidad pueda incidir y tener mayor participación en problemáticas que losafectan.

\section{METODOLOGÍA}

La investigación que se desarrolla es de tipo descriptivo, enfocada a identificar la realidad de la asociación Asoparibari, desde el punto de vista de capacidad organizacional como comunidad, empleando la herramienta cuantitativa Índice de Capacidades Organizacionales de Comunidades Cafeteras (ICOOC) desarrollada por Cenicafe. Así mismo, se desarrollará una encuesta a los potenciales beneficiarios del proyecto de construcción de la central de café cereza, mediante la cual se podrá cuantificar la viabilidad técnica para la construcción de la central. La investigación se efectuó con los integrantes de la asociación y posibles beneficiarios del proyecto, aplicando el Índice de Capacidad Organizacional e Índice de Viabilidad Técnica.

Además, teniendo en cuenta la metodología formulada por Cenicafé, es posible determinar el Índice de Viabilidad Técnica (IVT), que permitirá hacer una recomendación a la asociación de productores sobre la pertinencia de continuar o no con el proceso para la cons- 
trucción de una central de beneficio o hacer recomendaciones para mejorar los procesos organizacionales.

Para el cálculo de los índices, se realizó una encuesta a 74 caficultores en cada una de sus fincas. A estos caficultores se les informó previamente del proceso de investigación que llevó a cabo la Universidad Santo Tomás en el programa de Administración de Empresas Agropecuarias; el cual buscó generar resultados que permitieran proponer planes de acción para la Asociación en su proceso comunitario.

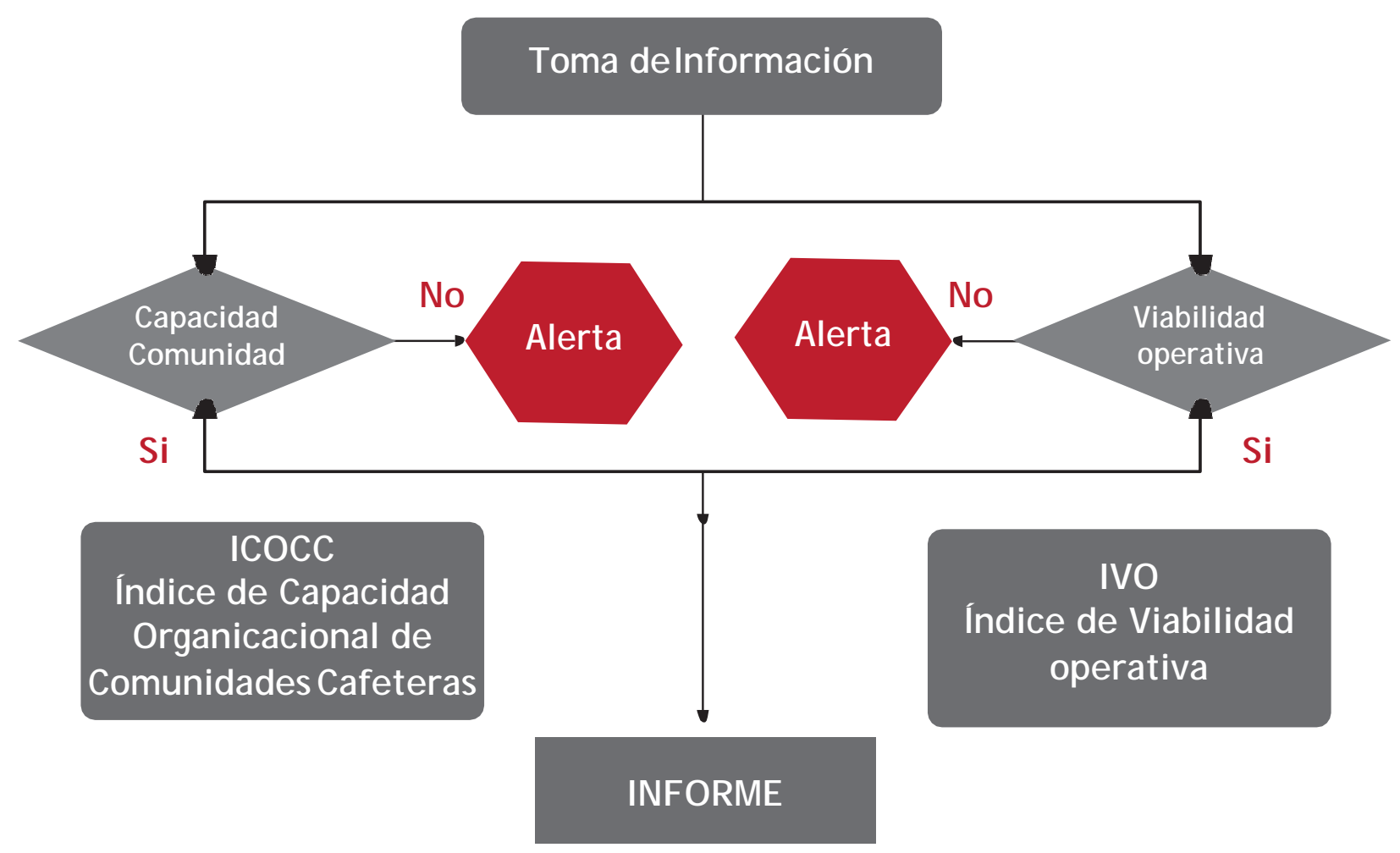

Figura 1. Diagrama del proceso de investigación Fuente: Cenicafé, 2016 
La población encuestada fue de 74 caficultores, de los cuales 33 son asociados hábiles, los 41 restantes son asociados no hábiles pero constituyen potenciales socios de la asociación. El análisis de los datos recolectados se realizó de dos formas, con un análisis estadístico y otro análisis con la metodología de Centro Nacional de Investigaciones del Café (Cenicafé), con este último se logró determinar los índices.

\section{RESULTADOS}

Tomando como base la metodología del Centro Nacional de Investigaciones del Café (Cenicafé) se aplicó el instrumento de Índice de Viabilidad Técnica IVT y el Índice de Capacidades Organizacionales de Comunidades Cafeteras ICOCC en la asociación Asoparibari del municipio de La Palma. Con esta aplicación de instrumentos se logró recolectar información trascendental de los beneficiarios de la posible Central de beneficio, la cual será insumo para justificar o rechazar la viabilidad del proyecto de laconstrucción de la Central de beneficio.

Con respecto al IVT, al construir la central de beneficio los costos de funcionamiento y mantenimiento serán menores, y los usuarios no tendrán que incurrir en costos adicionales en hacer arreglos a sus beneficiaderos y los que no lo poseen no tendrán la necesidad de construir uno. La disponibilidad de los servicios públicos básicos, como lo son el agua y la luz, en la central de beneficio de Asoparibariserá una oportunidad para suplir estas falencias, pues la central de benéfico contará con una planta eléctrica y el uso del agua será más reducido, lo cual también generará un impacto positivo en el medio ambiente. Esto también se verá reflejado en una disminución en los costos de servicios.

Al procesar el café en diferentes beneficiaderos los procesos son diferentes obteniendo heterogeneidad en la calidad del producto. Al usar la planta de beneficio los procesos se estandarizan y de esta forma el porcentaje de mayor calidad del producto aumentaría, obteniendo así mayoresbeneficios.

El $98.63 \%$ de los encuestados están interesado en aprovechar la pulpa y la miel del lavado de café. Esto se debe a que el manejo en las fincas es bastante difícil y costoso, además se puede convertir en una oportunidad económica para los asociados en la central de beneficio. Con el mucilago del café se pueden generar unos productos secundarios que representan beneficio económico para los productores. Adicional a esto, el uso de la miel que se extrae del fruto de café reducirá el impacto ambiental en la zona. Al construir la planta de beneficio se podrán sacar estos productos secundarios que generarán ingresos adicionales. 
El 100\% de los asociados confía en que tendrán una valoración de su café cereza que cumpla sus expectativas. Esta confianza de todos los productores indica que el proyecto de construcción de la central les traerá múltiples beneficios.

El $97.22 \%$ de los asociados a Asoparibari tiene la disponibilidad de tener una participación en las actividades de la asociación, esto se completa con la confianza que tienen en cuanto a que su producción será bien valorada. Este interés de la comunidad en la central de beneficio es evidente con estos resultados.

El $66 \%$ de los encuestados que tiene beneficiadero no cuentan con tratamiento de aguas residuales, lo cual genera un impacto ambiental negativo en la zona. La cantidad de agua usada en el beneficio de café se reducirá al construir la central de beneficio; además, al agua residual se le hará un tratamiento para mitigar el efecto en el ambiente. Así mismo, la confianza en la asociación hace que los asociados estén dispuestos a entregar su producto en la asociación para el benéfico del café en cereza.

Al procesar los datos con la metodología de Cenicafé, Índice de Viabilidad Técnica IVT, se obtuvo un resultado concordante con el análisis estadístico que se describió anteriormente. Con la metodología el Índice de Viabilidad
Técnica los resultados son muy favorables de prefactibilidad para la construcción de una central de café cereza en el municipio de la Palma, Cundinamarca. Los resultados están por rangos en categorías de bajo, medio, alto y muy alto.

La mayoría de usuarios se encuentra en el rango alto, lo cual evidencia que este estudio de prefactibilidad para la construcción de la central de beneficio es viable y los usuarios de Asoparibari tienen una viabilidad óptima para que puedan hacer uso de la central de beneficio y que sus condiciones como productores pueden mejorar.

Por último está el rango muy alto, el cual señala que la central de beneficio definitivamente mejorará las actividades de este grupo caficultores y, por ende, su calidad de vida. Este resultado permite recomendar a la asociación de productores Asoparibari la pertinencia de continuar con el proceso para la construcción de una central de beneficio.

Siendo la capacidad organizacional un factor determinante en una organización comunitaria, se han hecho diversos esfuerzos que permitan medirlo. El Ministerio de Agriculturay Desarrollo Rural, en su publicación Instrumento de diagnóstico del estado organizativo de comunidades campesinas locales, propone la 
medición de la capacidad organizacional a través de dos ámbitos: el interno u organizacional y el externo o institucional (2003).

Teniendo en cuenta lo anterior, esta investigación identificó el potencial que tiene la asociación Asoparibari tanto en términos técnico como organizacional, razón por la cual se utilizó la herramienta Índice de Capacidades Organizacionales de Comunidades Cafeteras ICOCC, con el fin de identificar fortalezas y debilidades de la asociación, desde el punto de vista de la organización, y de esta manera proponer planes de mejora.

Una vez aplicado el instrumento a los caficultores incluidos en este proyecto se concluye que los componentes junta administradora, asambleas y reuniones tienen una alta calificación, lo cual indica que la mayoría de los entrevistados ha participado de las asambleas y reuniones. Además poseen algún grado de conocimiento sobre la junta administradora $y$ el grado de confianza en los directivos. Por otra parte, el componente participación tiene una calificación media indicando que la participación de los asociados en las actividades de la asociación es media.

En el componente capacidad instalada, la Asociación Asoparibari se encuentra con una calificación dentro del rango bajo, lo cual indica que los encuestados tienen claro que no existe una sede propia y que los recursos con los que se dispone no son suficientes para operar. El componente recursos se encuentra en el rango medio, pues existe la participación de los asociados con dinero propio y de otras entidades; sin embargo, este componente podría ser mejor si se gestionan más proyectos y se implementan actividades que generen sostenibilidad económica para laasociación.

El componente servicios financieros y comerciales tiene una calificación baja, lo cual se debe a que la asociación no ofrece este tipo de servicios a sus asociados. En cambio, los componentes servicios de capacitación y asistencia, así como atención, presentan una calificación media, lo que indica que por medio de la asociación se han prestado servicios de capacitación en diferentes proyectos tanto a asociados como a no asociados, aunque se podrían prestar mejores servicios.

Algunos componentes de este índice, por ejemplo dinámicas, equidad de género, relaciones interpersonales y proyección, poseen una calificación en el rango medio; solamente confianza y acción colectiva se encuentran en el rango bajo. Ese último resultado es preocupante, ya que se relaciona con el apoyo que pueden dar los asociados cuando alguien se encuentra en una situación difícil o a cuántas personas acudiría para solventar una necesidad. Con este 
resultado se interpreta que no existe confianza en las demás personas y que ante situaciones difíciles los socios del grupo no cuentan con apoyo. Los demás componentes están en el rango medio, con un alto potencial para mejorar.

El resultado general del Índice de Capacidad Organizacional de la Asociación Asoparibari es de 0.69 , ubicándolo en el rango medio de calificación. Esto indica que la asociación tiene un alto potencial para mejorar y que en cada uno de sus componentes se deben formular planes de acción que permitan una mayor articulación entre sus asociados. También permitirá generar unos objetivos estratégicos que permitan a la asociación ofrecer mejores servicios, tanto a los asociados como a la comunidad en general, manteniendo en el tiempo una sostenibilidad económica y social. Este resultado ICOCC permitirá orientar esfuerzos para contribuir a que las acciones que se emprendan sean pertinentes ysostenibles.

\section{DISCUSIÓN Y PROPUESTA}

Este análisis ha implantado una metodología de gestión en el sector caficultor que permitehacer un estudio de prefactibilidad, antes de desarrollar nuevos proyectos, para evitar malgastar recursos económicos, que afectan a la población de caficultores, generando falsas expectativas $y$ la pérdida de credibilidad en las asociaciones.
El proyecto genera un análisis de prefactibilidad de la pertinencia para la construcción de una central de beneficio de café cereza, basado en el estudio de una organización de agricultores, además de proponer mejoras en la gestión y administración de proyectos comunitarios. Además, la Viabilidad Técnica - IVT de beneficiadero de café comunitario, como gestión de un proyecto social para una posibleconstrucción, teniendo en cuenta particulares. De esta forma se mejorará la Capacidad Tecnológica de los caficultores delmunicipio.

El estudio prefactibilidad de la construcción y operación de esta infraestructura se articula con la apuesta de la caficultura colombiana por ser rentable y $100 \%$ sostenible, teniendo en cuenta aspectos como:

- Solamente se debe producir buen café en cereza

\section{- Se tiene mayor control sobre el proceso} (consistencia)

- Se tiene mayor control sobre el impacto ambiental

- Mejor costo de oportunidad para los caficultores 
La investigación permitió evaluar la viabilidad para la creación de una Central Agroindustrial de Beneficio Húmedo de Café, que permitirá a los caficultores ahorrar hasta $10 \%$ en costos de producción por hectárea y optimizar la calidad del café con un beneficio $100 \%$ ecológico (cero contaminaciones) y menores costos en mano de obra, energía y agua

Se propone que la Asociación objeto de estudio construya la Central de Benéfico de una central de café cereza en el municipio de la Palma, Cundinamarca, dando un Enfoque de Desarrollo Humano, fortalecimiento de capacidades. También este modelo brinda un aprovechamiento de la economía de escala.

\section{CONCLUSIONES}

El estado de los beneficiaderos en las fincas, en su gran mayoría, es regular, malo o no existe; además no se cuenta con infraestructura para el manejo de los subproductos del café, tales como: aguas resultantes del proceso de poscosecha y la pulpa. Esto implica que para mejorar los beneficiaderos en cada una de las fincas se deberían hacer grandes inversiones, las cuales se podrían optimizar con la construcción de una central de café cereza. Por otra parte, el tema de la calidad del producto café pergamino seco es muy heterogéneo debido al mal estado de la infraestructura y a las diferencias en procesos de beneficio que se presentan en cada una de las fincas de los caficultores.

Por medio de la implementación de una central de café cereza, los procesos de poscosecha relacionados con el beneficio húmedo y secado del café se pueden estandarizar y lograr producir volúmenes de café pergamino seco con consistencia en sus características físicas y organolépticas. Con la puesta en marcha de una central de café cereza se puede hacer uso de una planta eléctrica, para los momentos en los cuales el servicio eléctrico falle en la zona.

Un alto número de las personas que fueron encuestadas manifestó interés en que le compraran el café en cereza, siendo este un elemento diferenciador a la hora de tomar una decisión en cuento a la viabilidad de una central de beneficio de café. Este elemento es clave debido a que en Cundinamarca son muy escasos los ejemplos de centrales de beneficio de café comunitarias, y por lo tanto se creía que habría alguna resistencia en torno a vender café cereza y no café seco como se ha realizado durante la mayor parte del tiempo.

La confiabilidad que tiene los productores en la asociación es favorable, pues además la mayoría están dispuestos a participar en el funcionamiento, aportando cada uno desde su 
experiencia y experticia, seguramente logrando mejores resultados y otras proyecciones de la asociación. Esta participación comunitaria puede ser aprovechada para obtener productos derivados del beneficio del café comouna alternativa adicional de generar ingresos para los asociados.

El cálculo del Índice de Viabilidad Técnica IVT está en un rango de viabilidad alto, indicando que se justifica la construcción de la central de beneficio. En este caso es necesario revisar algunos caficultores evaluados en la viabilidad técnica que tienen una baja calificación para recomendarles que continúen o no en el proyecto.

Finalmente, el ICOCC que la asociación está en una calificación media desde el punto de vista de su capacidad organizacional. Este es un factor que se debe evaluar al momento de definir el operador de la central de beneficio ya que se plantea que sea la asociación quien administre un modelo de negocio ubicado en el municipio de la Palma, sin embargo, se deben implementar previamente planes de mejora que permitan mejorar la situación económica, los servicios ofrecidos, la capacidad de liderazgo, el trabajo colectivo y la confianza entre los mismos socios.

\section{REFERENCIAS}

Arcila Pulgarin, J., Farfán Valencia, F., Moreno Berrocal,

A., Salazar Gutiérrez, L., \& Hincapié Gomez,

E. (2007). Sistemas de produccion de café en

Colombia. Chinchina:Cenicafe.

Centro Nacional de Investigaciones del Cafe - Cenicafé. (2016). cenicafe.org. Obtenido de www.cenicafe.org

DANE. (I 5 de 07 de 2017). Obtenido de http://www. dane.gov.co/index.php/estadisticas-por-tema/ agropecuario

Espinel, C. (2010). Índice ICO: Competencias Organizacionales Diagnóstico Organizacional Participativo, Rápido y Eficiente, Guía para facilitar su aplicación- USAID, Acción Social. Obtenido de En: http://www.ard.org.co/midas/departamentos/agricultores-y-cade- nasde-valor/pdf/Indice_ICO_ultimo.pdf.

Ministerio de Agricultura y Desarrollo Rural. (2003). Minagricultura. Obtenidodehttp://207.239.25 I. I 10:8080/ jspui/bitstream/I I 348/6369/ //I58.pdf

Urueña Gómez, M. (20|3). El mercado mundial y nacional del café en el siglo XXI. En Manual del cafetero colombiano: Investigacion y tecnologia para la sostenibilidad de la caficultura. Tomo I (págs. 17-25). Chinchina: FNC: Cenicafé. 